\title{
Metabolome and transcriptome analyses reveal quality change in the orange-rooted Salvia miltiorrhiza (Danshen) from cultivated field
}

\section{Zhilai Zhan}

National Resource Center for Chinese Materia Medica

\section{Wentao Fang}

National Resoure Center for Chinese Materia Medica

\section{Xiaohui Ma}

Yunnan University of Traditional Chinese Medicine

\section{Tong Chen}

National Resource Center for Chinese Materia Medica

\section{Guanghong Cui}

National Resource Center for Chinese Materia Medica

\section{Ying Ma}

National Resouce Center for Chinese Materia Medica

\section{Liping Kang}

National Resource Center for Chinese Matieria Medica

\section{Tiegui Nan}

National Resource Center for Chinese Materia Medica

Huixin Lin

National Resource Center for Chinese Materia Medica Jinfu Tang

National Resource Center for Chinese Materia Medica

\section{Yan Zhang}

National Resource Center for Chinese Materia Medica

Changjiangsheng Lai

National Resource Center for Chinese Materia Medica

\section{Zhenli Ren}

Shandong Danhong Pharmaceutical Co.

\section{Yanan Wang}

National Resource Center for Chinese Materia Medica

\section{Yujun Zhao}

National Resource Center for Chinese Materia Medica

Ye Shen 
National Resource Center for Chinese Materia Medica

\section{Ling Wang}

National Resource Center for Chinese Materia Medica

\section{Wen Zeng}

National Resource Center for Chinese Materia Medica

Juan Guo ( $\square$ guojuanzy@163.com )

China Academy of Chinese Medical Sciences

\section{Luqi Huang}

National Resource Center for Chinese Materia Medica

\section{Research article}

Keywords: Salvia miltiorrhiza Bunge (Danshen); tanshinones; metabolome profiling; mRNA expression profiling; ER-associated degradation

Posted Date: May 24th, 2019

DOI: https://doi.org/10.21203/rs.2.9771/v1

License: (c) (7) This work is licensed under a Creative Commons Attribution 4.0 International License. Read Full License

Version of Record: A version of this preprint was published at Chinese Medicine on October 2nd, 2019. See the published version at https://doi.org/10.1186/s13020-019-0265-6. 


\section{Abstract}

Backgroud The dry root of Salvia miltiorrhiza Bunge, or Danshen, is a well-known, traditional Chinese medicine. Tanshinones are active compounds that accumulate in the periderm, resulting in red-colored roots. However, lines with orange roots have been observed in cultivated fields. Here, we performed metabolome and transcriptome analyses to investigate the changes of orange-rooted Danshen. Results In total, 40 lipophilic components were detected by ultra-high-performance liquid chromatographyquadrupole time-of-flight mass spectrometry (UPLC/Q-Tof-MS). Seven compounds were significantly decreased in the orange Danshen, including the most abundant active compounds, tanshinone IIA and tanshinone I in normal Danshen. Systematic analysis of transcriptome profiles revealed that the downregulated genes related to catalytic dehydrogenation was not detected. However, two genes related to stress resistance, and four genes related to endoplasmic reticulum (ER)-associated degradation of proteins were up-regulated in orange Danshen. Conclusion Decreases in the content of dehydrogenated furan ring tanshinones such as tanshinone IIA resulted in phenotypic changes and quality degradation of Danshen. Transcriptome analysis indicated that incorrect folding and ER-associated degradation of corresponding enzymes, which could catalyze $\mathrm{C} 15-\mathrm{C} 16$ dehydrogenase, contributed to the decrease in dehydrogenated furan ring tanshinones, rather than lower expression of the relative genes.

\section{Background}

Danshen, the dry root and rhizome of Salvia miltiorrhiza Bunge, is one of the most versatile components in traditional Chinese medicine. More than 636 medicinal preparations utilize Danshen as their main constituent, which can be found in the Chinese patent medicine prescription library. Among them, Danshen Tablets and Fufang Danshen Dripping Pills have been widely used in modern Chinese clinical trials and are considered effective in treating cardiovascular and cerebrovascular diseases [1].

The main components of Danshen include hydrophilic phenolic acids and lipophilic diterpenoid tanshinones, which are used to evaluate the quality of medicinal materials [ 1,2$]$. Modern pharmacological studies and chemical investigations suggest that both components contribute to Danshen's pharmacological and therapeutic effects. Phenolic acid compounds are distributed in the roots and leaves of $S$. miltiorrhiza, while tanshinones mainly accumulate in the roots and rhizomes. More than 40 structurally diverse tanshinones have been isolated and identified since their discovery in the 1930s. Among them, tanshinone IIA, cryptotanshinone, tanshinone I, and dihydrotanshinone I are the main active ingredients (Fig. 1B) [ 3 ]. Most tanshinones have colors ranging from orange to red (Fig. 1B), which elicit the reddish phenotype of Danshen. These compounds have been extensively investigated for their wellknown cardiovascular activities, as well as for their anti-cancer activities in vitro and in vivo [ 4 ]. Tanshinone IIA has also been reported to have potential for treating human inflammation [ 5 ], as well as anti-adipogenic effects on 3T3-L1 cells and in zebrafish [ 6 ].

In China, more than 70,000 tons of Danshen is consumed every year. Before the 1970 s, wild Danshen was the main ingredient in traditional Chinese medicine. However, with increased demand, wild resources have 
greatly decreased. Now, Danshen is cultivated in northern and middle China, providing almost all the material for traditional patent prescriptions and for clinical use in Chinese medicine. Thus, the quality and consistency of cultivated Danshen are essential for medicinal security. Interestingly, some orange-rooted lines have appeared during cultivation, suggesting that variations in tanshinone content or composition may affect the phenotype and quality of Danshen (Fig. 1A). Additionally, this phenomenon is not an isolated case and has been observed in several different cultivation areas, including the Shandong, Henan, and Shanxi Provinces. Therefore, extensive investigation of the orange lines found in cultivated fields is important for further utilization of Danshen.

The genome sequence and transcriptome analysis of Danshen from various development periods [ 7-9 ], different tissues [ 10 ], and tissue cultures from different induction times were taken [11], which provide a foundation for the biological analysis of this useful medicinal plant. Based on these analyses, the biosynthesis and regulation of tanshinones and phenolic acids have made great strides [ 12-14 ]. However, there are still many problems that need to be studied based on comparative omics data and genome sequencing.

In this study, we utilized ultra-high-performance liquid chromatography-quadrupole time-of-flight mass spectrometry (UPLC/Q-TOF-MS) to analyze the component variations in both red (normal) and orangerooted Danshen to assess their differences in quality. In order to determine a possible explanation for these phenotypic changes, high-throughput RNA sequencing was performed to detect differences in gene expression between the two types of Danshen.

\section{Results}

\section{PCA and OPLS-DA results}

Lipophilic diterpenoid tanshinone compounds can be detected with high sensitivity in positive ion (ESI $\left.{ }^{+}\right)$ mode. Thus, UPLC/Q-TOF-MS in the ESI ${ }^{+}$mode was employed to analyze the metabolites profiling of normal and orange Danshen. As can be seen in the total ion chromatogram, some peaks decreased or almost disappeared in the orange Danshen tanshinones (Fig. 1B). Progenesis QI was utilized for data pretreatment to generate a convenient dataset containing variables and observations.

First, MS data of samples in the ESI ${ }^{+}$mode were statistically analyzed by untargeted principal component analysis (PCA), which was utilized to determine the similarity of the metabolite profiles among the fractions and retention time. Exact mass and ion intensity were used as variables. Points in the PCA score plot represent data observations; the closer the points, the more similar the data. Samples of normal and orange Danshen were well clustered and segregated into two different groups (Fig. 2A).

To determine the best discrimination of normal and orange Danshen and to discover the most important contributing variables, supervised orthogonal partial least-squares discriminant analysis (OPLS-DA) was employed. A scatter plot (S-plot) was obtained from the loading of the OPLS-DA score plot, which 
highlighted the variables most responsible for differences among the groups (Fig. 2B). Each data point is an exact mass/retention time pair and corresponds to a marker ion. Based on the OPLS-DA analysis (Fig. 2B), 10 potential markers were detected and found to be higher in normal Danshen than in orange (Fig. 2C). This indicates that the compound with a retention time of $11.41 \mathrm{~min}$ (i.e., tanshinone IIA) contributed to the most variation between the two groups.

\section{MS data analysis of the decreased tanshinones}

Fragment ions of lipophilic diterpenoids were primarily derived from the losses of $\mathrm{H}_{2} \mathrm{O}$ (18 Da), $\mathrm{CH}_{3}(15$ $\mathrm{Da})$, and $\mathrm{CO}(28 \mathrm{Da})$. In total, 40 compounds were identified by comparing their exact molecular weight and MS fragmentation with the standard compounds or data found in published papers (Table S1) [ 1518]. Seven compounds were detected and found to decrease in orange Danshen. After comparison to the standard compounds, six were identified, including tanshindiol C ( $R T=1.21 \mathrm{~min})$, tanshindiol B ( $R T=1.54$ min), tanshinol B (RT = $2.71 \mathrm{~min})$, tanshinone IIB ( $R T=3.56 \mathrm{~min})$, tanshinone (I RT = $8.94 \mathrm{~min})$, and tanshinone IIA (RT = $11.44 \mathrm{~min}$ ). Additionally, the compound with $\mathrm{RT}=10.35 \mathrm{~min}$ was identified as $1,2-$ didehydrotanshinone IIA based on its exact molecular weight and MS fragmentation, based on the data reported by Yang et al. [16].

Although the confirmed compounds had distinct structures, they shared the same dehydrogenated furan ring. Specifically, tanshinone IIA and tanshinone I with long conjugated bonds were the terminal biosynthesis products of tanshinones and had a deep red color (Fig. 1B). The decrease in tanshinone IIA content appears to lead to root color change from red to orange. It is proposed that tanshinone IIA was converted from cryptotanshinone by dehydrogenation (Fig. 3). Tanshinone IIA could also be used as a substrate for the formation of tanshinone IIB. Meanwhile, tanshinone I was supposed to be converted from dihydrotanshinone I by dehydrogenation. This indicates that the reduction in tanshinone IIA, tanshinone IIB, and tanshinone I may be due to the decreased expression of or degradation of enzymes that catalytically dehydrogenate substrates to products.

\section{Transcriptome profiling and expression of known functional genes in the biopathways of tanshinones}

In total, 189 million clean reads were generated for all samples, where $33,172,046,31,992,708$, and $29,460,962$ were found in the three normal Danshen samples and 33,163,130,31,364,922, and 30,122,148 were found in the three orange Danshen samples. The reference transcriptome assembled by using pooled reads contained 36,017 unigenes longer than $500 \mathrm{nt}$. Unigenes were searched against the NCBI Nr and Nt, KEGG, SwissProt, Pfam, and GO databases, and it was found that approximately $88.10 \%$ of the unigenes could be annotated in at least one of the databases. A total of 18,114 ( 50\%) unigenes could be annotated in KEGG, with 350 unigenes involved in the metabolism of terpenoids and polyketides, 108 involved in the biosynthesis of the terpenoid backbone, and 34 involved in diterpenoid biosynthesis (Fig. 4). 
Tanshinones are abietane-type norditerpenoid quinone natural products. Diterpenoids have been reported to originate in the MEP pathway of plastids, however, cross-talk does occur between the MVA and MEP pathways during the biosynthesis of tanshinones [ 19, 20]. Genes involved in the biosynthesis of the terpenoid backbone (i.e., IPP and DMAPP) have been investigated in normal and orange Danshen. From the common diterpenoid precursor (i.e., geranylgeranyl diphosphate (GGPP)) to the metabolic intermediates of tanshinones, five genes are responsible for tanshinone biosynthesis and have been characterized, including two diterpenoid synthases (i.e., SmCPS1 and SmKSL1) [ 21, 22 ], as well as three cytochrome P450s (i.e., CYP76AH1, CYP76AH3, and CYP76AK1) [ 23,24 ]. In this study, the expression of upstream genes in normal and orange Danshen did not differ significantly (Fig. 3). Additionally, in the metabolic profiling there was no significant difference observed in the biopathway intermediates, including cryptotanshinone, dihydrotanshinone, and the intermediate sugiol. This indicates that there is a balanced metabolic flow upstream.

\section{KEGG enrichment analysis of down-regulated genes}

In total, 104 genes were significantly expressed in normal and orange Danshen (with adjust $p$ value < 0.05 ; Table S2). In order to understand which categories are overrepresented, differentially expressed genes (DEGs) were further analyzed by a KEGG enrichment analysis. In total, there were 58 downregulated genes in orange Danshen. The KEGG pathway analysis mapped 10 categories (Fig. 5), including cyanoamino acid metabolism, peroxisome, the AMP-activated protein kinase (AMPK) signaling pathway, and plant-pathogen interactions (Table S3). The annotated function of these genes, based on other databases, included glutamyltranspeptidase, glutathione hydrolase, peptidylprolyl isomerase, hydroxy fatty acid phosphatase, and epoxide hydrolase activities (Table S2).

Metabolic profiling revealed that compounds with $\mathrm{C}_{15}-\mathrm{C}_{16}$ double bonds in the furan ring were the principal structures found in normal and orange Danshen. Most dehydrogenation reactions were catalyzed by cytochrome P450s, NADP/NAD-dependent dehydrogenases, and reductases, thus, we selected these gene families for further analysis. Additionally, down-regulated genes related to reductase or dehydrogenase catalytic activity that were highly expressed in normal Danshen were also selected. However, there were no genes related to dehydrogenation with expression profiles concordant with the accumulation of tanshinone IIA or tanshinone I. Therefore, genes that were up-regulated in orange Danshen were the focus.

\section{KEGG enrichment analysis of up-regulated genes}

There were 46 genes that were up-regulated in orange Danshen based on the DESeq analysis. In total, 16 significant KEGG categories were identified, including protein processing in the endoplasmic reticulum (ER), RNA transport, and protein export (Fig. 5 and Table S3). Interestingly, four genes were assigned to the "protein processing in the ER." In the up-regulated genes, these four genes were annotated as BIPs (i.e., c80749_g1, up-regulated by 5.2 fold; and c80749_g2, up-regulated by 5.9 fold), heat shock protein 70 
kDa (i.e., c91931_g1, whose read count value was up-regulated from 0 to 112.5), and a molecular chaperone (i.e., c75139_g1, up-regulated by 2.6 fold).

In the ER, correctly folded proteins are packaged into transport vesicles, while incorrectly folded proteins are retained in the complex with molecular chaperones and bind to BIP, then are directed towards degradation, which is mediated by heat shock proteins and molecular chaperones [25]. The four genes related to ER-associated protein degradation were up-regulated in orange Danshen, while there was no identifiable down-regulated gene responsible for catalytic $\mathrm{C}_{15}-\mathrm{C}_{16}$ dehydrogenation, which indicates that there is an alternative mechanism related to the decrease of tanshinone IIA content. Based on the transcriptome analysis, it appears that the low accumulation of tanshinone IIA and related tanshinones with dehydrogenated furan rings may be a result of the degradation of corresponding enzymes that may catalyze $\mathrm{C}_{15}-\mathrm{C}_{16}$ dehydrogenase, rather than the lower expression of relative genes.

In addition to the genes assigned to protein processing in the ER, there was one gene annotated as a zincfinger protein and two genes annotated as eukaryotic translation initiation factor 5B (elF5B) were upregulated. Zinc-finger proteins have been previously reported to play a central role in abiotic stress resistant $[26,27]$. As for elF5B, it is one of two initiation factors that is universally conserved in prokaryotes, archaea, and eukaryotes. In Arabidopsis thaliana, it has been reported to play a role in biological or abiotic stress [ 28 ]. Up-regulation of zinc-fingers and elF5B indicates that orange Danshen may have experienced stress during cultivation.

\section{Discussion}

The conjugated system of tanshinones results in a red phenotype in Danshen. In this study, during resource investigation, plants with orange roots were found to occur in a different cultivation base, suggesting that the concentration or constituents of tanshinones in orange Danshen may differ from normal Danshen. Compared to normal Danshen, the UPLC/Q-TOF-MS analysis revealed that the peaks of tanshinone IIA and tanshinone I almost disappeared. In total, seven tanshinones with dehydrogenated furan rings were decreased in orange Danshen. Tanshinone IIA with long conjugated bonds had a deep red color, accounting for $25.3 \%-63.4 \%$ of the total tanshinones and are the terminal products of tanshinone biosynthesis [15]. Low concentration of tanshinone IIA and related tanshinones, such as tanshinone $\mathrm{I}$, with a double $\mathrm{C}_{15}-\mathrm{C}_{16}$ bond led to an orange phenotype in cultivated fields.

Tanshinone IIA, cryptotashinone, tanshinone I, and dihydrotanshinone I are index components of Danshen [29]. Specifically, tanshinone IIA is an indicative lipophilic ingredient in the Pharmacopoeia of the People's Republic of China [1]. Sodium sulfonate forms of tanshinone IIA have been widely used to treat coronary heart disease, angina pectoris, and myocardial infarction in the clinical setting [30]. Thus, the decreased content of tanshinone IIA and other related compounds would detrimentally affect the quality of Danshen and its utilization. 
Decreased expression of or the degradation of enzymes involved in catalytic dehydrogenation may result in low transformation efficiency of tanshinones I and IIA from dihydrotanshinone I and cryptotanshinone, respectively. However, there was not a gene detected, which was responsible for catalytic dehydrogenation in the down-regulated genes of orange Danshen based on the comparative transcriptome analysis. However, two genes have been reported to be related to stress [ 26-28, 31], and four genes related to ER-associated protein degradation [25], which were found to be significantly upregulated in orange-Danshen.

Zinc-finger proteins have been reported to play a central role in abiotic stress resistance, especially oxidative stress in Arabidopsis, during which the expression of both zinc-finger and heat-shock proteins increased [ 26, 27]. The elF5B mutant of Arabidopsis behaves as the wild type in the absence of stress, although it is more temperature sensitive, which indicates that elF5B plays a role in stress resistance [28]. Moreover, it interacts with other initiation factors and GTP to initiate translation, and has been reported to have the ability to prevent thermal aggregation and promote refolding of heat-labile proteins, such as chaperone-like activity in Pisum sativum [ 31 ]. It was proposed that Danshen with orange roots might be suffered from stress. This resulted in unusual initiation of genes responsible for catalytic dehydrogenation, which might further result in uncorrected folding of the corresponding proteins. The uncorrected folding proteins were then guided to ER-associated protein degradation, and finally interrupted the dehydrogenation step in biopathway of tanshinones.

\section{Conclusions}

In this study, metabolome and transcriptome analyses were integrated to analyze the orange Danshen from cultivated fields. Metabolome analyses indicated that low concentration of tanshinone IIA and related tanshinones, such as tanshinone $\mathrm{I}$, with a double $\mathrm{C}_{15}-\mathrm{C}_{16}$ bond resulted an orange phenotype of Danshen in cultivated fields. During cultivation, both biotic and abiotic stressors are actively present throughout the growth period of Danshen. Up-regulated zinc-finger proteins and elF5s indicate stress resistance during cultivation. Danshen roots that experience stress may result in unusual transcription initiation and incorrect protein folding, which may lead to the degradation of proteins related to the dehydrogenation of tanshinones in $\mathrm{C}_{15}-\mathrm{C}_{16}$ bonds. This limited dehydrogenation of cryptotanshinone and dihydrotanshinone I into tanshinones IIA and I products, respectively, leads to a reduced quality of Danshen in cultivated fields. Future studies should functionally characterize the exact genes involved in catalytic dehydrogenation of furan rings, and investigate the degradation mechanism and incorrect folding of catalytic enzymes that depend on zinc-finger proteins, heat-shock proteins, and elF. In conclusion, because quality degradation often takes place during cultivation [32], the data presented here and the proposed mechanism in this study will provide a reference for the cultivation of Chinese material medicine.

\section{Materials And Methods}

Plant materials 
Danshen were collected from the Chinese herbal medicine cultivation base located in Changqing, Shandong Province, China. Three independent normal samples and three independent orange samples were collected for metabolite profiling. An additional three samples of each type of Danshen were collected for transcriptome analysis during the florescence period in July. At this time, growth conditions transfer from vegetative to reproductive growth and secondary metabolites are accumulated during this period. Plant materials were frozen with liquid nitrogen and stored at $-80^{\circ} \mathrm{C}$ for subsequent metabolome and RNA-sequencing analyses.

\section{Chemicals and reagents}

Reference standards of tanshinone IIA, cryptotanshinone, tanshinone I, dihydrotanshinone I, tanshinone IIB, dihydroisotanshinone I, danshenxinkun B, methylenetanshinquinone, tetrahydrotanshinone I, miltirone, dehydromiltirone, sageone, isocryptotanshinone, tanshindiol A, tanshindiol B, tanshindiol C, 1,2dihydrotanshinquinone, tanshinol B, sugiol, and methyltanshinonate (Beijing Rongchengxinde Technology Development Co., Ltd, Beijing, China) had purities above $95 \%$.

Acetonitrile and methanol (Merck, Darmstadt, Germany), as well as formic acid (Fisher Scientific, Geel, Belgium), were HPLC grade. Ultra-pure water $(18.2 \mathrm{M} \Omega / \mathrm{cm})$ was purified using a Barnstead GenPure UV/UF water purification system (Thermo Fisher Scientific, Langenselbold, Germany).

\section{Sample and standard preparation}

Danshen roots used for metabolome analysis were dried in the shade, and then pulverized and sieved through a 50 mesh. Powdered samples $(1.0 \mathrm{~g})$ were dissolved in $10 \mathrm{~mL}$ of $80 \%$ methanol and ultrasonically extracted for $30 \mathrm{~min}$ at $25^{\circ} \mathrm{C}$ and $40 \mathrm{kHz}$ (SCIENT ultrasonic processor, Ningbo, China). After centrifugation at $13,000 \mathrm{rpm}$ for $10 \mathrm{~min}$, supernatants were filtered through a $0.22 \mu \mathrm{m}$ membrane filter. In order to identify the compounds in S. miltiorrhiza samples, mixed standard solutions (100 $\mu \mathrm{g} / \mathrm{mL}$ ) were made by weighing and dissolving each reference standard in methanol.

\section{Metabolite profiling by UPLC/Q-TOF-MS}

Metabolite profiling was performed on a UPLC system equipped with a high-pressure pump, automatic sample manager, and column heater (Waters Acquity, USA). A UPLC BEH C18 Column $(2.1 \mathrm{~mm} \times 50 \mathrm{~mm}$, $1.7 \mu \mathrm{m})$ (Waters Acquity) and a C18 pre-column $(2.1 \mathrm{~mm} \times 5 \mathrm{~mm}, 1.7 \mu \mathrm{m})$ (Waters Acquity) were used. The mobile phase consisted of water with $0.1 \%(\mathrm{v} / \mathrm{v})$ formic acid $(A)$ and acetonitrile with $0.1 \%(\mathrm{v} / \mathrm{v})$ formic acid (B). The gradient program was as follows: 0-1.5 min, 35-40\% B; 1.5-7.0 min, 40-50\% B; 7.010.0 min, 50-58\% B; 10.0-11.8 min, 58-80\% B; 11.8-12.3 min, 80-98\% B; 12.3-14.0 min, 98\% B; 14.0-14.5 $\min , 98-30 \% \mathrm{~B} ; 14.5-16.0 \mathrm{~min}, 30 \% \mathrm{~B}$. The injection volume was $1 \mu \mathrm{L}$. The target column temperature was $40^{\circ} \mathrm{C}$. 
Chemical profile analysis was performed on a Xevo G2-S Q-Tof-MS analyzer (Waters Micromass, Manchester, UK) operated in both positive electrospray ionization modes. In the $\mathrm{ESI}^{+}$mode, parameters were set as follows: mass range, 50-1500 Da; collision energy, 35-60 V; capillary voltage, $0.5 \mathrm{kV}$; cone voltage, $40 \mathrm{~V}$; source temperature, $100^{\circ} \mathrm{C}$; desolvation gas temperature, $450^{\circ} \mathrm{C}$; cone gas flow rate, $50 \mathrm{~L} / \mathrm{h}$; desolvation flow rate, $900 \mathrm{~L} / \mathrm{h}$.

\section{Data pretreatment and statistical analyses}

The centroid MS raw data were analyzed using the Progenesis QI 2.1 software (Waters, Milford, USA) for biomarker discovery. Multiple adduct ions, including $[\mathrm{M}+\mathrm{H}]^{+},[\mathrm{M}+\mathrm{Na}]^{+},[2 \mathrm{M}+\mathrm{Na}]^{+},[\mathrm{M}+\mathrm{K}]^{+},[\mathrm{M}+\mathrm{H}-\mathrm{H} 2 \mathrm{O}]^{+}$, and $[\mathrm{M}+\mathrm{NH} 4]^{+}$, were selected or self-edited to remove redundant adduct ion species. PCA and OPLS-DA were conducted in order to determine differences in the metabolic composition of normal and orange Danshen. The total ions of the samples were exported by Progenesis QI 2.1 software (Waters Co.).

\section{RNA sequencing, de novo transcriptome assembly, and analysis}

Total RNA was extracted from the main root samples utilizing the Trizol method (Invitrogen, California, USA). RNA sequencing was performed on an Illumina HiSeq platform (Novogene Bioinformatics Technology Co. Ltd, China) for paired-end reads of 150 200 bp. Raw data reads were first processed to remove adaptors and low-quality bases in order to obtain clean reads for downstream analysis. Left- and right-sequenced reads were concatenated separately and submitted to Trinity to assemble a reference transcriptome with default parameters [33]. Clean reads were then sequenced and aligned to the reference transcriptome by Bowtie2 [34]; read counts for each gene were computed by in-house scripts. All de novo assemblages were searched against the $\mathrm{NCBI} N r(E=1.0 \mathrm{E}-5)$ and $\mathrm{Nt}(\mathrm{E}=1.0 \mathrm{E}-5)$, Swiss-Prot $(\mathrm{E}=1.0 \mathrm{E}-5)$, and Pfam $(\mathrm{E}=1.0 \mathrm{E}-2)$ databases. The $\mathrm{KEGG}$ pathway analysis was conducted using the KEGG automatic annotation server $(E=1.0 \mathrm{E}-10)$.

\section{Analysis of differentially expressed genes}

The DEGs were analyzed using the DESeq2 R package v1.16.1 [35], which provides statistical procedures for determining differential expression using a model based on the negative binomial distribution. The $\mathrm{p}$ values were adjusted using the Benjamini-Hochberg procedure to control for the false discovery rate. Genes with an adjusted $p$-value $<0.05$ were considered to be differentially expressed. The KOBAS software was used to test the statistical enrichment of DEGs in the KEGG pathways [36].

\section{Acknowledgments}

The authors thank the Chinese herbal medicine cultivation base for providing Danshen material. 
Funding: This work was supported by grants from the National Natural Science Foundation of China (81403049, 81573532, 81822046), Key project at central government level: The ability to establish sustainable use of valuable Chinese medicine resources (2060302), National Key R\&D Program of China (2017YFC1700805, 2018YFD0201100), Development and Reform Commission Standardization Project (ZYBZH-C-QIN-46, ZYBZH-C-JL-25), Hunan Province Universities 2011 Collaborative Innovation Center for Protection and Utilization of Hu-xiang Chinese Medicine Resources.

Availability of data and material: All data generated or analysed during this study are included in this published article and its supplementary information files

Author Contributions: ZLZ, WTF, XHM, GHC, JG and LQH planed and desigened the research. ZLZ, WTF, $X H M$, and YM performed the experiments. ZLZ, WTF, TC, LPK, TGN, HXL, JFT, CJSL, YNW, YJZ, WZ, and JG analyzed the data. ZLZ, WTF, XHM, TC, YS, JG and LQH wrote the paper. Funding acquisition, ZLZ., $Y Z, L W, J G$ and LQH. ZLR and YZ provided the plant materials.

Ethics approval and consent to participate: Not applicable

Consent for publication: Not applicable

Competing interests: The authors declare no conflict of interest.

\section{References}

1. Pharmacopoeia NCoC: Pharmacopoeia of the People's Republic of China, 2015 ed. Beijing, China; 2015.

2. Wang BQ: Salvia miltiorrhiza: Chemical and pharmacological review of a medicinal plant. Journal of Medicinal Plants Research 2010, 4(25):2813-2820.

3. Zhong G-X, Li P, Zeng L-J, Guan J, Li D-Q, Li S-P: Chemical characteristics of Salvia miltiorrhiza (Danshen) collected from different locations in China. J Agric Food Chem 2009, 57(15):6879-6887.

4. Zhang Y, Jiang P, Ye M, Kim SH, Jiang C, Lu J: Tanshinones: sources, pharmacokinetics and anti-cancer activities. International Journal of Molecular Sciences 2012, 13(10):13621-13666.

5. Robertson AL, Holmes GR, Bojarczuk AN, Burgon J, Loynes CA, Chimen M, Sawtell AK, Hamza B, Willson J, Walmsley SR et al: A zebrafish compound screen reveals modulation of neutrophil reverse migration as an anti-inflammatory mechanism. Sci Transl Med 2014, 6(225):225-229.

6. Park Y-K, Obiang-Obounou BW, Lee J, Lee T-Y, Bae M-A, Hwang K-S, Lee K-B, Choi J-S, Jang B-C: AntiAdipogenic Effects on 3T3-L1 Cells and Zebrafish by Tanshinone IIA. International Journal of Molecular Sciences 2017, 18(10):2065. 
7. Xu H, Song J, Luo H, Zhang Y, Li Q, Zhu Y, Xu J, Li Y, Song C, Wang B et al: Analysis of the genome sequence of the medicinal plant Salvia miltiorrhiza. Mol Plant 2016, 9(6):949-952.

8. Zhang G, Tian Y, Zhang J, Shu L, Yang S, Wang W, Sheng J, Dong Y, Chen W: Hybrid de novo genome assembly of the Chinese herbal plant danshen (Salvia miltiorrhiza Bunge). Gigascience 2015, 4(62):1-4.

9. Hua W, Zhang Y, Song J, Zhao L, Wang Z: De novo transcriptome sequencing in Salvia miltiorrhiza to identify genes involved in the biosynthesis of active ingredients. Genomics 2011, 98(4):272-279.

10. Yang L, Ding G, Lin H, Cheng H, Kong Y, Wei Y, Fang X, Liu R, Wang L, Chen X et al: Transcriptome analysis of medicinal plant Salvia miltiorrhiza and identification of genes related to tanshinone biosynthesis. PLoS One 2013, 8(11):e80464.

11. Gao W, Sun H-X, Xiao H, Cui G, Hillwig M, Jackson A, Wang X, Shen Y, Zhao N, Zhang L et al: Combining metabolomics and transcriptomics to characterize tanshinone biosynthesis in Salvia miltiorrhiza. BMC Genomics 2014, 15(1):73.

12. Ma XH, Ma Y, Tang JF, He YL, Liu YC, Ma XJ, Shen Y, Cui GH, Lin HX, Rong QX et al: The biosynthetic pathways of tanshinones and phenolic acids in Salvia miltiorrhiza. Molecules 2015, 20(9):16235-16254.

13. Shi M, Luo X, Ju G, Li L, Huang S, Zhang T, Wang H, Kai G: Enhanced diterpene tanshinone accumulation and bioactivity of transgenic Salvia miltiorrhiza hairy roots by pathway engineering. J Agric Food Chem 2016, 64(12):2523-2530.

14. Yu H, Guo W, Yang D, Hou Z, Liang Z: Transcriptional profiles of SmWRKY family genes and their putative roles in the biosynthesis of tanshinone and phenolic acids in Salvia miltiorrhiza. International Journal of Molecular Sciences 2018, 19(6):1-17.

15. Xie W, Zhang H, Zeng J, Chen H, Zhao Z, Liang Z: Tissues-based chemical profiling and semiquantitative analysis of bioactive components in the root of Salvia miltiorrhiza Bunge by using laser microdissection system combined with UPLC-q-TOF-MS. Chemistry Centtral Journal 2016, 10(42):1-13.

16. Yang M, Liu A, Guan S, Sun J, Xu M, Guo D: Characterization of tanshinones in the roots of Salvia miltiorrhiza (Dan-shen) by high-performance liquid chromatography with electrospray ionization tandem mass spectrometry. Rapid Commun Mass Spectrom 2006, 20(8):1266-1280.

17. Yang ST, Wu X, Rui W, Guo J, Feng YF: UPLC/Q-TOF-MS analysis for identification of hydrophilic phenolics and lipophilic diterpenoids from Radix Salviae Miltiorrhizae. Acta Chromatographica 2015, 27(4):711-728.

18. Zhao Q, Song Z, Fang X, Pan Y, Guo L, Liu T, Wang J: Effect of genotype and environment on Salvia miltiorrhiza roots using LC/MS-based metabolomics. Molecules 2016, 21(4):1-17. 
19. Yang D, Du X, Liang X, Han R, Liang Z, Liu Y, Liu F, Zhao J: Different roles of the mevalonate and methylerythritol phosphate pathways in cell growth and tanshinone production of Salvia miltiorrhiza hairy roots. PLoS One 2012, 7(11):e46797.

20. Laule O, Furholz A, Chang H, Zhu T, Wang X, Heifetz P, Gruissem W, Lange M: Crosstalk between cytosolic and plastidial pathways of isoprenoid biosynthesis in Arabidopsis thaliana. Proc Natl Acad Sci USA 2003, 100(11):6866-6871.

21. Cui G, Duan L, Jin B, Qian J, Xue Z, Shen G, Snyder JH, Song J, Chen S, Huang L et al: Functional divergence of diterpene syntheses in the medicinal plant Salvia miltiorrhiza. Plant Physiol 2015, 169(3):1607-1618.

22. Gao W, Hillwig ML, Huang L, Cui G, Wang X, Kong J, Yang B, Peters RJ: A functional genomics approach to tanshinone biosynthesis provides stereochemical insights. Org Lett 2009, 11(22):5170-5173.

23. Guo J, Ma X, Cai Y, Ma Y, Zhan Z, Zhou YJ, Liu W, Guan M, Yang J, Cui G et al: Cytochrome P450 promiscuity leads to a bifurcating biosynthetic pathway for tanshinones. New Phytol 2016, 210(2):525534.

24. Guo J, Zhou YJ, Hillwig ML, Shen Y, Yang L, Wang Y, Zhang X, Liu W, Peters RJ, Chen X et al: CYP76AH1 catalyzes turnover of miltiradiene in tanshinones biosynthesis and enables heterologous production of ferruginol in yeasts. Proc Natl Acad Sci USA 2013, 110(29):12108-12113.

25. Stolz A, Wolf DH: Endoplasmic reticulum associated protein degradation: a chaperone assisted journey to hell. Biochimica et Biophysica Acta-Molecular Cell Reasearch 2010, 1803(6):694-705.

26. Davletova S, Schlauch K, Coutu J, Mittler R: The zinc-finger protein Zat12 plays a central role in reactive oxygen and abiotic stress signaling in Arabidopsis. Plant Physiol 2005, 139(2):847-856.

27. Rizhsky L, Davletova S, Liang H, Mittler R: The zinc finger protein Zat12 is required for cytosolic ascorbate peroxidase 1 expression during oxidative stress in Arabidopsis. The Journal of Biological Chemistry 2004, 279(12):11736-11743.

28. Zhang L, Liu X, Gaikwad K, Kou X, Wang F, Tian X, Xin M, Ni Z, Sun Q, Peng H et al: Mutations in elF5B confer thermosensitive and pleiotropic phenotypes via translation defects in Arabidopsis thaliana. The Plant Cell 2017, 29(8):1952-1969.

29. Hou JJ, Wu WY, Da J, Yao S, Long HL, Yang Z, Cai LY, Yang M, Liu X, Jiang BH et al: Ruggedness and robustness of conversion factors in method of simultaneous determination of multi-components with single reference standard. J Chromatogr 2011, 1218(33):5618-5627.

30. Zhang H, Long M, Wu Z, Han X, Yu Y: Sodium tanshinone IIA silate as an add-on therapy in patients with unstable angina pectoris. J Thorac Dis 2014, 6(12):1794-1799. 
31. Suragani M, Rasheedi S, Hasnain SE, Ehtesham NZ: The translation initiation factor, PelF5B, from Pisum sativum displays chaperone activity. Biochem Biophys Res Commun 2011, 414(2):390-396.

32. Wang TL, Guan W, Sun K, Wang S, Chi XL, Guo LP: Progress in researches on pathogens, epidemiology and integrated control of diseases on Salvia miltiorrhiza in China. China Journal of Chinese Materia Medica 2018, 43(11):2402-2406.

33. Grabherr MG, Haas BJ, Yassour M, Levin JZ, Thompson DA, Amit I, Adiconis X, Fan L, Raychowdhury $\mathrm{R}$, Zeng $\mathrm{Q}$ et al: Full-length transcriptome assembly from RNA-Seq data without a reference genome. Nat Biotechnol 2011, 29:644-652.

34. Langmead B, Salzberg SL: Fast gapped-read alignment with Bowtie 2. Nat Methods 2012, 9:357-365.

35. Love Ml, Huber W, Anders S: Moderated estimation of fold change and dispersion for RNA-seq data with DESeq2. Genome Biol 2014, 15(12):550-565.

36. Olyarchuk JG, Wei L, Cai T, Mao X: Automated genome annotation and pathway identification using the KEGG Orthology (KO) as a controlled vocabulary. Bioinformatics 2005, 21(19):3787-3793.

\section{Figures}
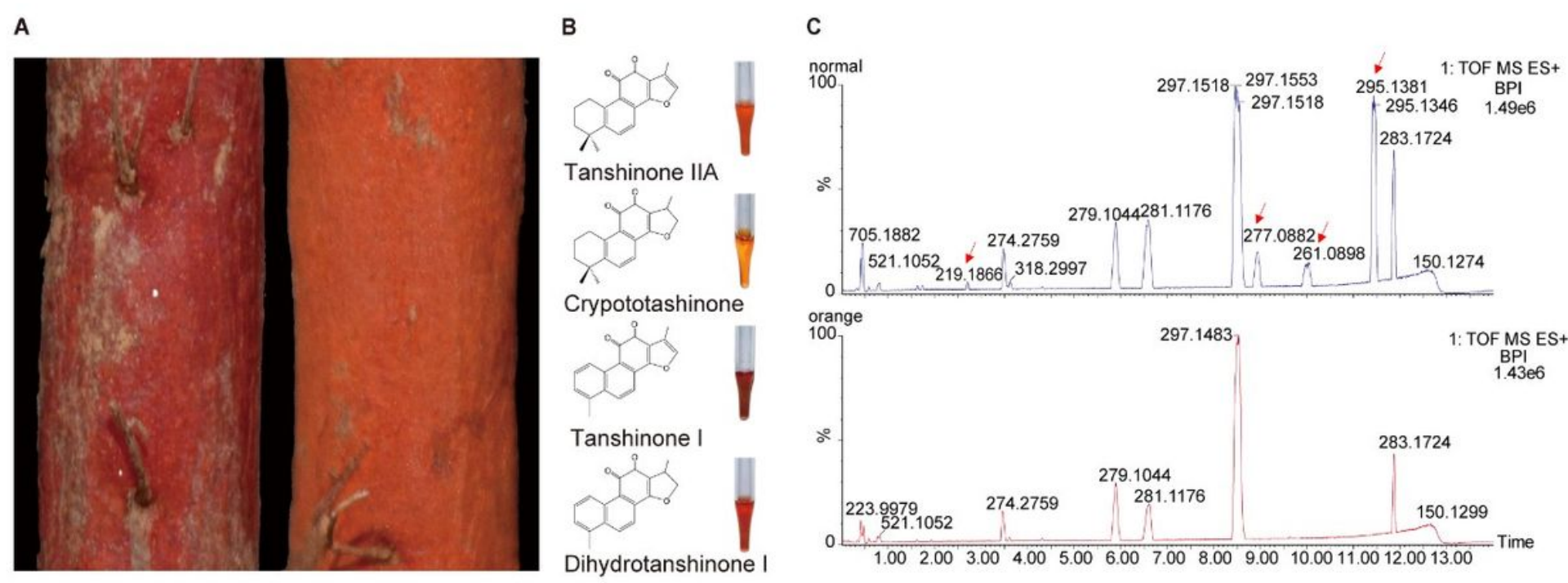

\section{Figure 1}

(A) Color phenotypes of normal Danshen (left) and orange Danshen (right). (B) Phenotypes of tanshinone IIA, cryptotashinone, tanshinone I, and dihydrotanshinone I. (C) The chromatogram of total ions found in normal Danshen (top) and orange Danshen (bottom). 

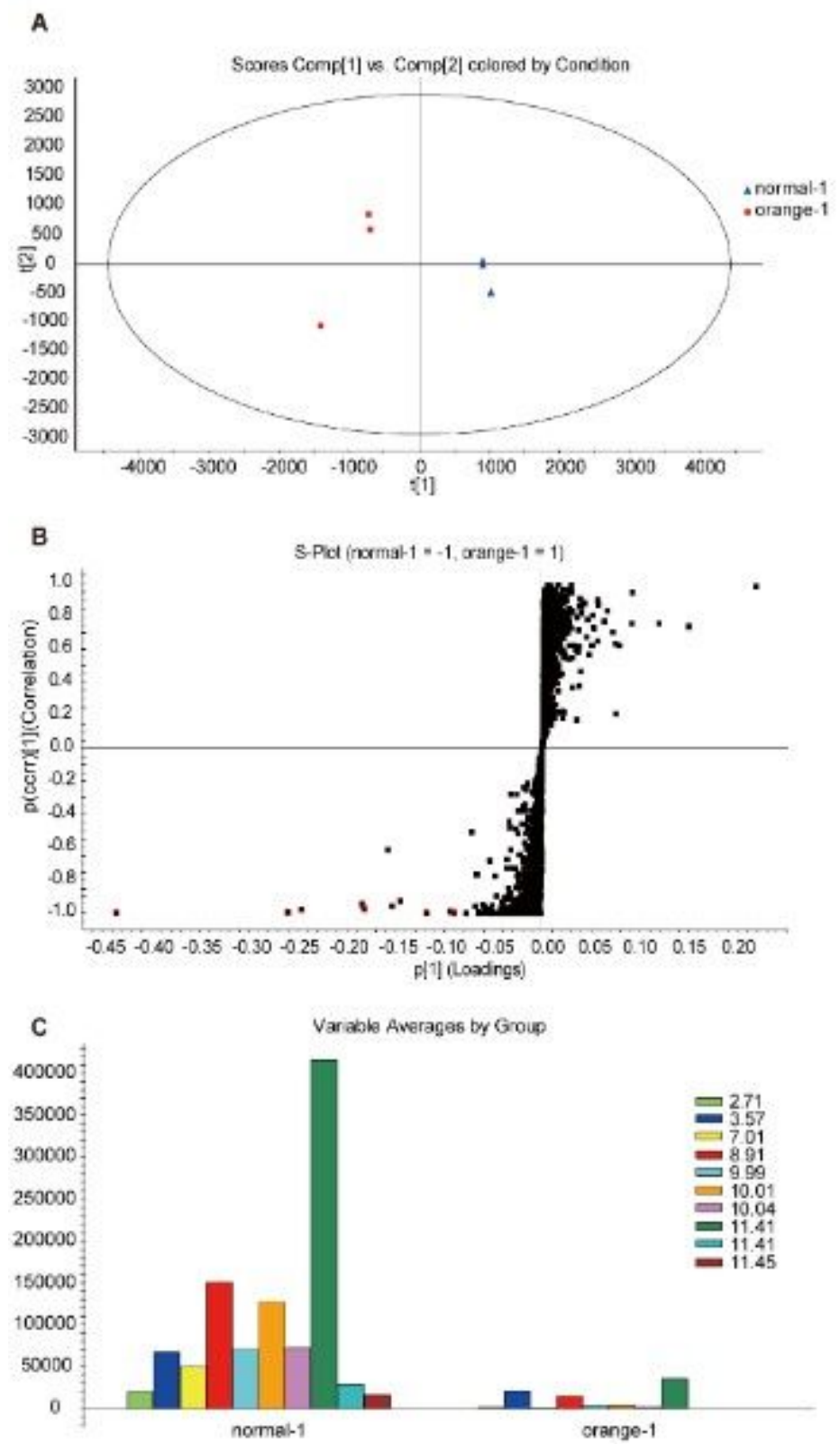

Figure 2

Chemometric analysis of normal and orange Danshen. (A) PCA analysis and score plot of normal and orange Danshen. (B) S-plot from the loading of the OPLS-DA. The x-axis indicates the variable contributions. The farther a data point lies from 0 , the more it contributes to the variance of the sample. The $y$-axis indicates the samples' correlations within the same sample group. The farther an exact mass/retention time pair lies from the value 0 , the better its correlation with the injections. (C) Variable averages of ten biomarkers in normal Danshen identified by OPLS-DA. 


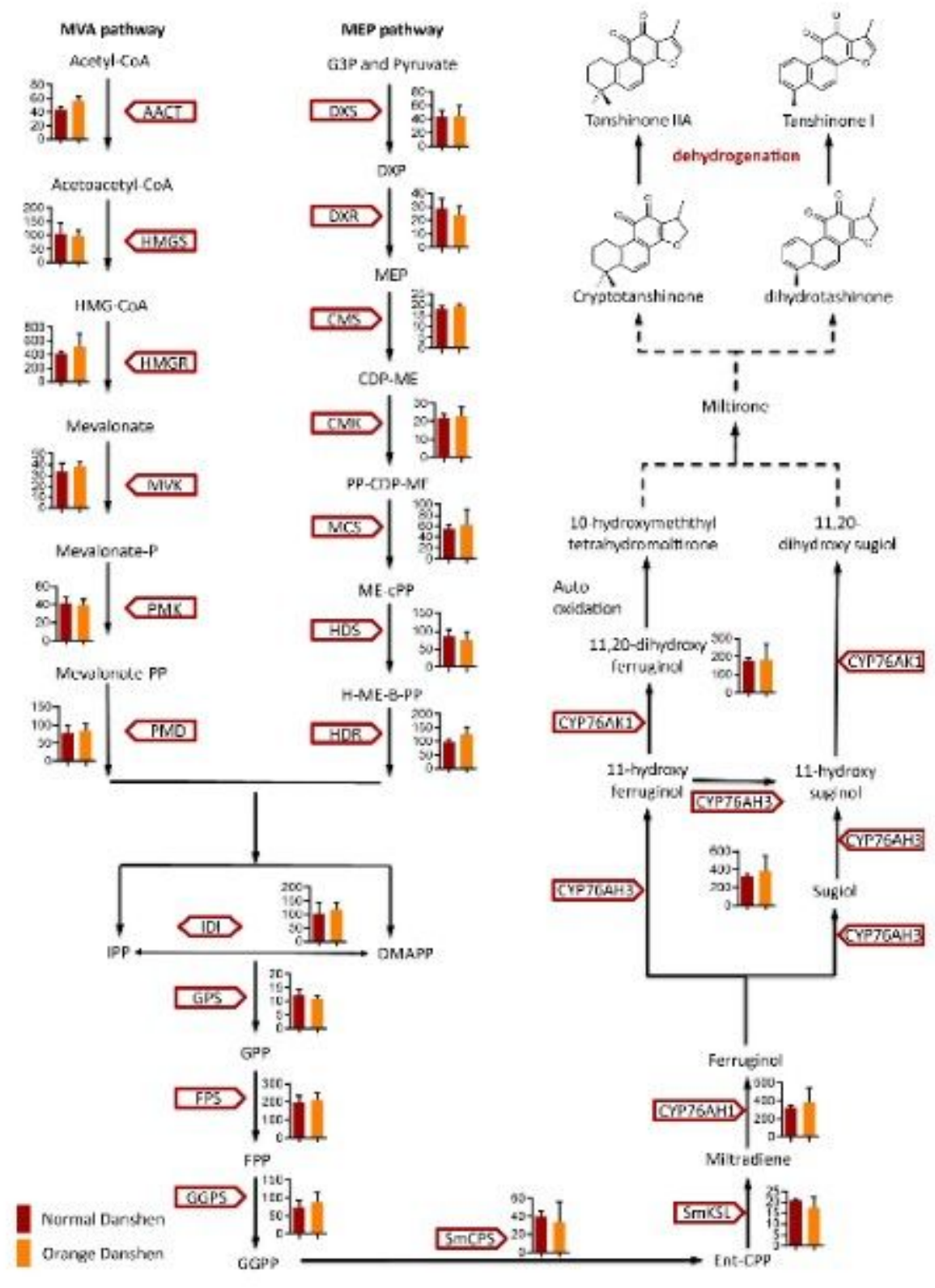

\section{Figure 3}

Expression of known functional genes involved in the biopathways of tanshinones. MEP pathway: the 2C-methyl-D-erythritol 4-phosphate pathway; MVA pathway: the mevalonate pathway. Acetoacetyl-CoA: Acetoacetyl coenzyme A; Acetyl-CoA: Acetyl coenzyme A; CDP-ME: 4-(Cytidine 5'-diphospho)-2-C-methylD-erythritol; DMAPP: Dimethylallyl pyrophosphate; DXP: 1-Deoxy-D-xylulose 5-phosphate; Ent-CPP: Entcopalyl diphosphate; FPP: Farnesyl diphosphate; G3P: Glyceraldehyde 3-phosphate; GGPP:

Geranylgeranyl diphosphate; GPP: Geranyl diphosphate; H-ME-B-PP: 1-Hydroxy-2-methyl-2-butenyl 4diphosphate; HMG-CoA: 3-Hydroxy-3-methylglutaryl CoA; IPP: Isopentenyl pyrophosphate; ME-cPP: 2-CMethyl-D-erythritol 2,4-cyclodiphosphate; MEP: 2-C-Methyl-D-erythritol 4-phosphate; Mevalonate-P: Mevalonate-5-phosphate; Mevalonate-PP: Mevalonate-5-diphosphomevalonate; MVA: Mevalonate; PPCDP-ME: 2-Phospho-4-(cytidine 5'-diphospho)-2-C-methyl-D-erythritol. AACT: Acetoacetyl-CoA transferase; CMK: 4-(Cytidine 5'-diphospho)-2-C-methyl-D-erythritol kinase; CMS: 4-(Cytidine 5'-diphospho)-2-C-methyl- 
D-erythritol synthase; DXR: 1-deoxy-D-xylulose-5-phosphate reductoisomerase; DXS: 1-deoxy-D-xylulose-5phosphate synthase; FPS: FPP synthase; GGPS: GGPP synthase; GO: GPS: GPP synthase; HDS: 4-

Hydroxy-3-methylbut-2-enyl diphosphate synthase; HMGR: 3-Hydroxy-3-methylglutaryl CoA reductase; HMGS: Hydroxymethylglutaryl-CoA synthase; IDI: Isopentenyl-diphosphate delta-isomerase; MCS: 2-CMethyl-D-erythritol 2,4-cyclodiphosphate synthase; MVK: Mevalonate kinase; PMD:

Diphosphomevalonate decarboxylase; PMK: Phosphomevalonate kinase; SmCPS: Salvia miltiorrhiza copalyl diphosphate synthase; SmKSL: Salvia miltiorrhiza kaurene synthase-like.

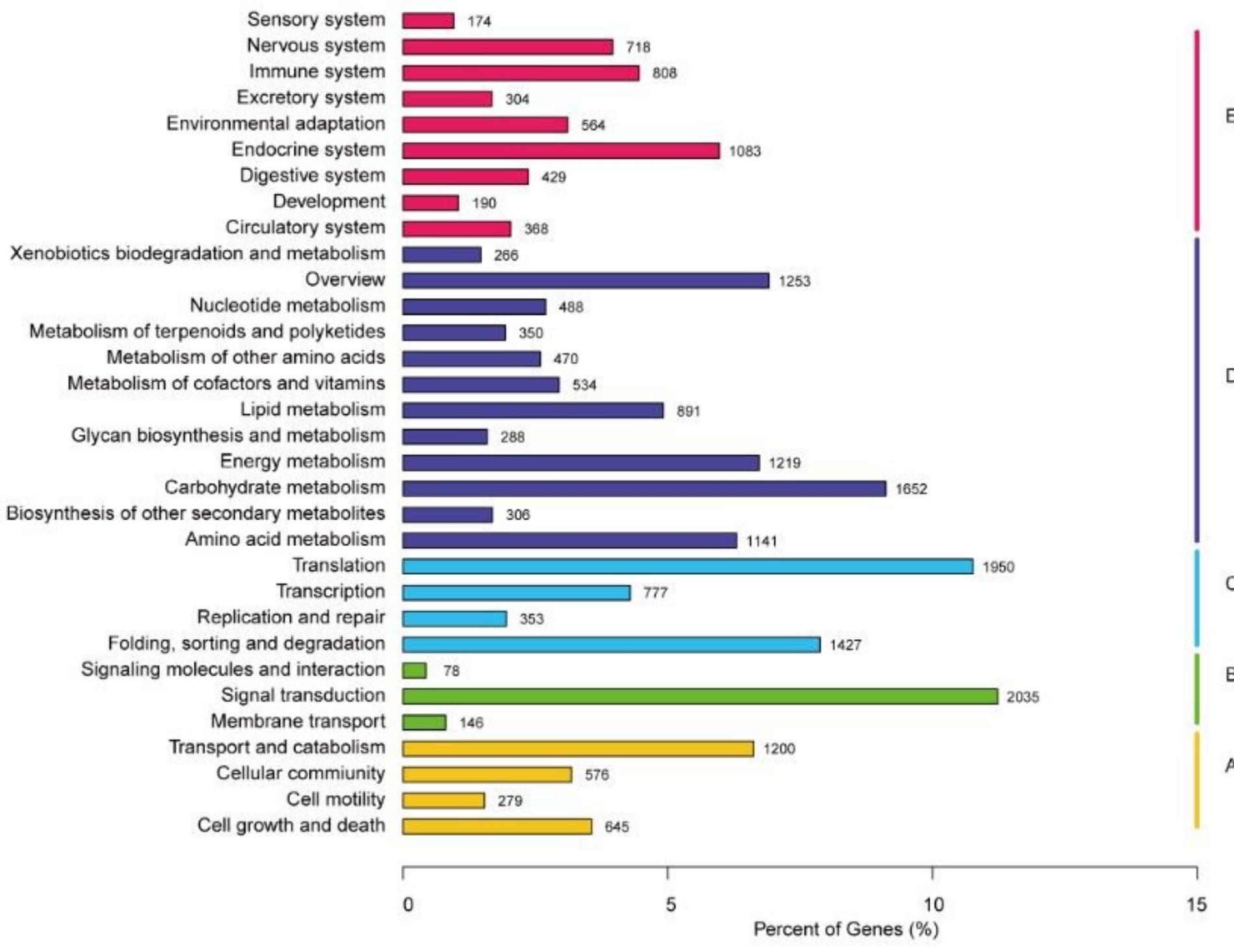

\section{Figure 4}

KEGG classification of the assembled unigenes. A: Cellular Processes; B: Environmental Information Processing; C: Genetic Information Processing; D: Metabolism; E: Organismal Systems. 


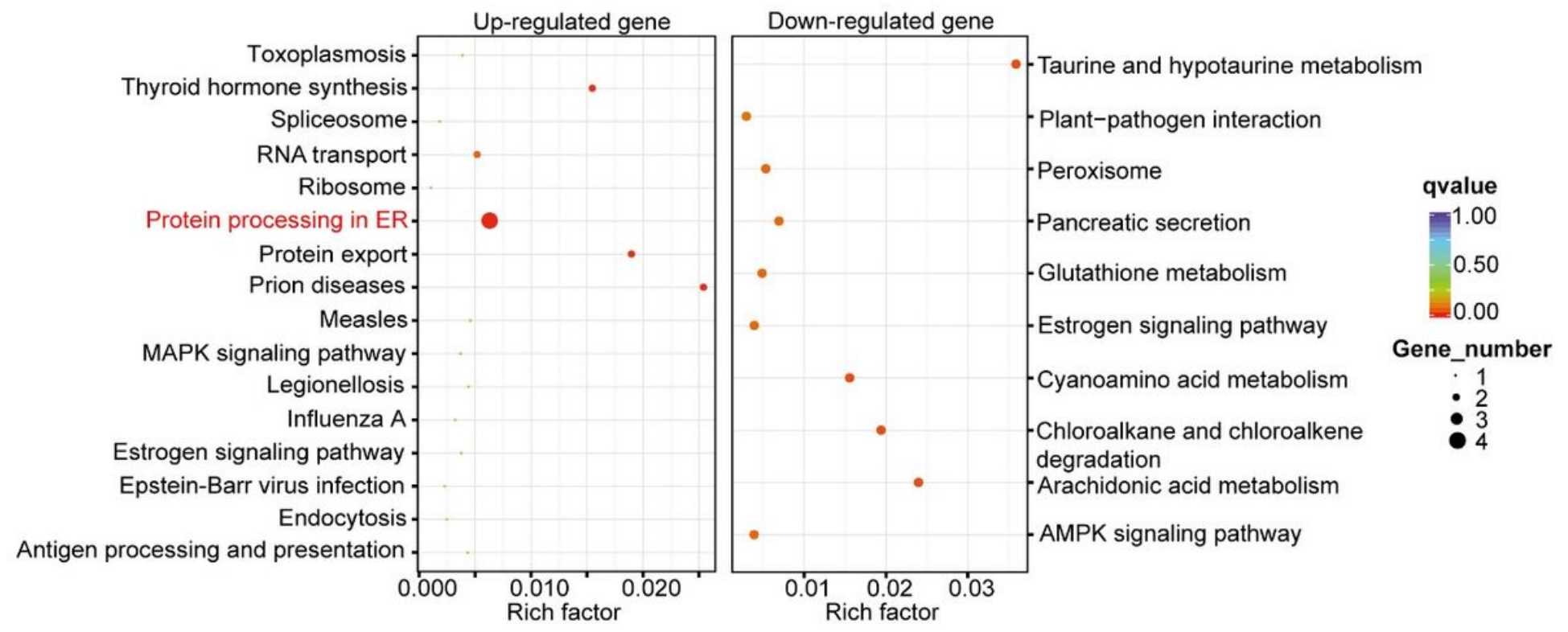

\section{Figure 5}

Scatterplot of the KEGG pathway of significantly enriched DEGs in orange Danshen. The rich factor refers to the ratio of the number of DEGs to the total number of genes enriched in a specific category.

\section{Supplementary Files}

This is a list of supplementary files associated with this preprint. Click to download.

- supplement1.xlsx

- supplement2.xIsx

- supplement2.docx 